\title{
Artigo \\ Projeto de uma Antena Planar Dipolo Curvado para Aplicação na Comunicação da Indústria 4.0
}

\author{
Estefanie Rayane S. de Oliveira ${ }^{[1]}$, Édson Fernandes C. Filho ${ }^{[2]}$, Matheus Emanuel T. Sousa ${ }^{[3]}$, \\ Humberto D. de Andrade ${ }^{[4]}$ e José Lucas da S. Paiva ${ }^{[5]}$ \\ ${ }^{[1]}$ Universidade Federal Rural do Semi-árido; estefanierayane159@ gmail.com \\ ${ }^{[2]}$ Universidade Federal Rural do Semi-árido; edson.fernandes.chaves.381@ gmail.com \\ ${ }^{[3]}$ Universidade Federal Rural do Semi-árido; matheus.sousa@ufersa.edu.br \\ ${ }^{[4]}$ Universidade Federal Rural do Semi-árido; humbertodionisio@ufersa.edu.br \\ ${ }^{[5]}$ Universidade Federal Rural do Semi-árido; jose.paiva@ufersa.edu.br \\ Recebido: 05/07/2020; \\ Aceito: 09/08/2020; \\ Publicado: 11/09/2020.
}

Resumo: O presente artigo contempla uma análise qualitativa do funcionamento de uma Antena Planar Dipolo Curvado, do tipo meander line e a sua aplicabilidade na Indústria 4.0. O termo Indústria 4.0 está diretamente relacionado com a Quarta Revolução Industrial, caracterizada pelo desenvolvimento tecnológico inteligente. Como por exemplo, o aperfeiçoamento de sistemas que promovem automação de tarefas, controle e compartilhamento de dados por meio de redes sem fio que possibilitam a tomada de decisões em tempo real. Tecnologias de destaque originadas em meio a esse período, como a Internet of Things (IoT), estão associadas a conectividade facilitada existente entre o mundo físico e digital por meio da internet ou de elementos miniaturizados, gerando otimização nos processos fabris e rotineiros. Particularmente, as antenas planares se destacam nesse quesito, por proporcionarem a transmissão ou recepção de sinais por meio de ondas eletromagnéticas, apresentarem baixo custo de fabricação, pequeno volume e peso. O objetivo proposto foi projetar, simular e analisar uma antena meander line que opere na faixa de frequência ISM (Industrial, Scientific and Medical) 2,4GHz, verificando sua eficácia para utilização na comunicação da Indústria 4.0. Com o auxílio do software HFSS®, foram obtidos resultados satisfatórios a respeito da frequência de ressonância e diagrama de radiação do dispositivo projetado, o qual alcançou um ganho máximo de -10dB.

Palavras-chave: meander line; Internet of Things; faixa de frequência ISM.

Abstract: This article contemplates a qualitative analysis of the operation of a Curved Dipole Planar Antenna, of the meander line type and its applicability in Industry 4.0. The term Industry 4.0 is directly related to the Fourth Industrial Revolution, characterized by intelligent technological development. As for example, the improvement of systems that promote automation of tasks, control and data sharing through wireless networks that enable decision making in real time. Outstanding technologies originating in the middle of this period, such as the Internet of Things (IoT), are associated with the facilitated connectivity existing between the physical and digital world through the internet or miniaturized elements, generating optimization in the manufacturing and routine processes. Particularly, planar antennas stand out in this regard, as they provide the transmission or reception of signals by means of electromagnetic waves, have low manufacturing cost, small volume and weight. The proposed objective was to design, simulate and analyze a meander line antenna that operates in the $2.4 \mathrm{GHz}$ ISM (Industrial, Scientific and Medical) frequency range, verifying its effectiveness for use in Industry 4.0 communication. With the aid of the HFSS® software, satisfactory results were obtained regarding the resonance frequency and radiation diagram of the designed device, which reached a maximum gain of $-10 \mathrm{~dB}$.

Key-words: meander line; Internet of Things; ISM frequency range. 


\section{INTRODUÇÃO}

$\mathrm{A}$ o longo dos séculos a sociedade sofreu profundas transformações em decorrência das revoluções industriais e suas inovações. Primeiramente com o desenvolvimento da linha de montagem, depois com a utilização da eletricidade, e em seguida, com o surgimento da tecnologia da informação, invenções essas que marcaram a Primeira, a Segunda e a Terceira Revolução Industrial, respectivamente. Além disso, desde o início do século XXI até os dias atuais, vive-se uma Quarta Revolução Industrial, conhecida como Indústria 4.0, que incrementa inteligência à tecnologia existente a fim de melhorar e otimizar a sociedade, a economia e a maneira de viver, a qual sempre se encontra em constantes mudanças. Pode-se citar como exemplos de tecnologias deste período, a impressão 3D, a inteligência artificial e a automação residencial e fabril. Uma tecnologia de destaque, que avança continuamente e está diretamente associada a Indústria 4.0 é a Internet das Coisas, ou Internet of Things (IoT), cujo conceito segundo [1], pode ser entendido como um ambiente de objetos físicos conectados à internet, entre si e com os usuários, por meio de sensores pequenos e embutidos, que introduzem soluções funcionais nos processos do dia a dia.

Desse modo, as transformações ocorridas durante a Quarta Revolução Industrial são responsáveis por criar um mundo mais inteligente e conectado, visando conforto, praticidade, produtividade e compartilhamento rápido de informações. Essa realidade de conexão facilitada é possível em virtude de elementos presentes no interior dos dispositivos voltados para telecomunicação, como as antenas, componentes fundamentais de um sistema de transmissão que utiliza o espaço livre como meio de transmissão. Nesse sentido, as antenas têm sido largamente comercializadas, sendo que um dos tipos de antena bastante utilizado em receptores e transmissores, são as antenas Planares Dipolo Curvado (Meander Line Antennas - MLA).

A tecnologia das antenas melhora significativamente a performance de sistemas baseado em redes sem fio, com altos valores de qualidade de sinal e uma maior capacidade de cobertura de transmissão. As particularidades da MLA a tornam uma potencial alternativa para aplicações diversas na Indústria 4.0, tecnologia que frequentemente demanda novos serviços para comunicação móvel que se adequem facilmente às necessidades. Dessa forma, este artigo tem como objetivo projetar, simular e analisar o funcionamento de uma antena Planar Dipolo Curvado do tipo meander line, operando com frequência dentro da faixa ISM $2,4 \mathrm{GHz}$ para aplicações industriais, médicas e científicas. O estudo irá contemplar a interpretação dos resultados relacionados ao ganho, perda de retorno, largura de banda e diagramas de radiação de campo elétrico e magnético da antena, no intuito de verificar seu desempenho e a eficácia de sua aplicabilidade na comunicação da Indústria 4.0.

\section{REFERENCIAL TEÓRICO}

\subsection{Indústria 4.0}

O crescimento tecnológico que avança continuamente vêm transformando os paradigmas da indústria tradicional. Nesse contexto, a Indústria 4.0 agrega máquinas inteligentes, análise computacional avançada e trabalho colaborativo entre pessoas conectadas, proporcionando eficiência operacional ao longo de toda a cadeia de produção e logística [2]. A conectividade é possibilitada por sistemas capazes de promover a comunicação autônoma dos dispositivos eletrônicos entre si e com os usuários. Essa autonomia nos processos proporciona a troca de informações em tempo real de modo preciso, aumento na produtividade em tempo reduzido, monitoramento de todo o processo produtivo e maior segurança na produção.

Desse modo, a Indústria 4.0 engloba o uso de algumas tendências tecnológicas para automação e troca de dados, além de utilizar conceitos de Sistemas Ciber-físicos e Internet das Coisas, e ainda, tecnologias como robótica, impressão 3D, realidade aumentada, realidade virtual e inteligência artificial. Em particular, a Internet das Coisas se destaca por estar associada ao alto nível de conectividade existente entre o mundo físico e digital e desenvolver novas formas de consumo, comunicação e conduta. A IoT está presente nos variados sistemas utilizados pelos setores agropecuário e de transporte aéreo e automotivo por exemplo, permitindo um maior potencial operacional com reconhecimento facial, uso de robôs, serviços de GPS e aplicativos que auxiliam na coleta de dados e informações. Logo, a IoT é de fundamental importância para os conceitos de Indústria 4.0 devido a possibilidade de conexão sem fio por meio de sensores e dispositivos eletrônicos, que permitem a centralização e automação do controle de produção e atividades do cotidiano, promovendo melhorias contínuas e eficiência nos processos característicos de uma indústria inteligente. 
A conectividade entre os dispositivos móveis é estabelecida por padrões que operam em faixas de frequências específicas dependendo do país ou região. Por exemplo, o padrão GSM (Global System for Mobile Communications) ou segunda geração $(2 \mathrm{G})$, precursor na transferência de dados e na transmissão de forma digital que utiliza as faixas de frequência entre 900MHz e 1800MHz. Outro exemplo, é o UMTS (Universal Mobile Telecommunications System) ou terceira geração (3G), que possibilita um uso efetivo de banda larga móvel e opera nas faixas de frequência de $900 \mathrm{MHz}$ e $2100 \mathrm{MHz}$. A tecnologia LTE (Long Term Evolution) ou quarta geração (4G) é mais moderna e veloz e utiliza a banda de frequência de $700 \mathrm{MHz}$ e $2500 \mathrm{MHz}$ dependendo de cada operadora. Além disso, uma banda de frequência bastante utilizada para aplicações em rede sem fio é a ISM (Industrial, Scientific and Medical) $2,4 \mathrm{GHz}$, faixa não licenciada que a comunidade científica utiliza para projetar e realizar testes e ensaios. Essa faixa compreende as frequências de $2400 \mathrm{MHz}$ até $2483,5 \mathrm{MHz}$, em que operam o padrão 802.11 b desenvolvido pela IEEE. São definidos 11 canais para utilização do padrão ISM que compreendem frequências entre a mais baixa de $2,401 \mathrm{GHz}$ e a mais alta com $2,473 \mathrm{GHz}$. Um exemplo desse padrão é o Wireless Local Area Network (WLAN), que permite criar redes de pequeno alcance com potência controlada, como o WIFI.

\subsection{Antena Meander Line}

O IEEE Standard Definitions of Terms for Antennas (IEEE Std 145 - 1973) define uma antena como um meio pelo qual uma onda eletromagnética pode ser radiada para o espaço livre ou captada e transferida para uma linha de transmissão [3]. Particularmente, a antena planar dipolo curvado do tipo meander line (MLA), também conhecida como antena de linha dobrada, é uma antena plana amplamente utilizada devido suas características adaptativas a superfícies curvas ou planas e por serem discretas, simples e de baixo custo de fabricação. Segundo [4], essas antenas consistem em uma plaqueta (ou patch) metálica disposta sobre um lado de um substrato, composto por material dielétrico, e um plano terra do outro lado do substrato. A plaqueta pode possuir diferentes formatos, seja quadrada, circular ou retangular por exemplo, dependendo da finalidade do projeto. O modelo e o dimensionamento adequado é o que irá tornar os sistemas mais ou menos confiáveis.

A antena do tipo meander line ilustrada na Figura 1 é composta por um fio dobrado continuamente, destinado a reduzir o comprimento ressonante [5] e possui como configuração mais comum a de meandros quadrados. Nessa configuração, o comprimento da plaqueta irradiante é encurtado dobrando o condutor em ângulos retos $\left(90^{\circ}\right)$ e formando um modelo de patch em formato de zigzag, cuja quantidade de voltas (meandros) é determinada de acordo com as características do projeto. Dessa forma, sua utilização é eficaz para aplicações em altas frequências de ressonância e em espaços pequenos, um exemplo prático de sua utilização é o Bluetooth em dispositivos móveis comerciais.

Por se tratar de uma antena planar, a MLA geralmente apresenta largura de banda estreita, mas isso pode ser melhorado com aplicação do uso de técnicas associadas já devidas na literatura. Segundo [4], métodos como aumentar a espessura do substrato e utilizar material com constante dielétrica de valor baixo podem elevar a largura de banda da antena. No estudo proposto por [6], duas antenas slot meander patch (SPM) são projetadas e confeccionadas para aplicação em dispositivos portáteis da tecnologia 4G LTE (Long Term Evolution). Ambas divergem apenas na atribuição da largura do meandro, sendo que a antena multibanda com larguras desiguais de meandros apresentou maior largura de banda e eficiência de radiação em comparação com a antena convencional de meandros iguais. Contudo, observou-se que a largura de banda também é melhorada a partir de ajustes na largura dos meandros sem alterar outros parâmetros.

A largura de banda (Band Width - BW) de uma antena é definida como a faixa de frequência na qual o desempenho da antena, referido a algumas características, atende um padrão especificado [4]. Assim, refere-se ao intervalo entre a frequência inferior e superior em valor máximo de perda de retorno de $-10 \mathrm{~dB}$, em torno da frequência central de operação. Há também, a largura de banda fracionária dada em porcentagem e calculada pela Equação (1), que indica a classificação da banda da antena, sendo que quanto maior, mais abrangente será a recepção e transmissão de sinal [7]:

$$
B W \%=2 \frac{f_{\text {superior }}-f_{\text {inferior }}}{f_{\text {superior }}+f_{\text {inferior }}} \times 100 \%
$$

sendo classificada em:

- Banda estreita: $0 \%<B W \%<1 \%$

- Banda larga: $1 \% \leq B W \% \leq 20 \%$

- Banda ultra larga: $B W \% \geq 20 \%$ 
Com relação aos valores de perda de retorno $(R L)$, segundo [3] são obtidos por meio da Equação (2), onde $\mathrm{S}(1,1)$ se refere ao parâmetro de espalhamento, também conhecido como coeficiente de reflexão. E para que a antena ressoe e funcione efetivamente, a perda de retorno medida deve apresentar valor menor ou igual a $-10 \mathrm{~dB}$.

$$
R L=-20 \log \left|S_{11}\right|
$$

As antenas do tipo meander line são utilizadas em diversas aplicações, sendo projetadas com materiais, modelos e dimensões distintas a depender da necessidade. Um exemplo é o estudo desenvolvido por [8], que projetou e avaliou o desempenho de uma antena meander line eletricamente pequena e com guia de onda coplanar para operar na frequência de $2,5 \mathrm{GHz}$ em tecnologias LTE (Long Term Evolution). O design e simulação da antena foram realizados no HFSS, utilizando um substrato Rogers Ultralam $1250 \mathrm{com} 2 \mathrm{~mm}$ de espessura e permissividade relativa de 2,5 que resultou em um ganho eficientemente alto de 7,2dB e perda de retorno de $-20 \mathrm{~dB}$. A pesquisa realizada por [9], por sua vez, propõe o projeto, simulação e fabricação de uma antena meander line para aplicação na comunicação dispositivo a dispositivo (D2D) na frequência VHF (Very High Frequency). Os resultados obtidos na simulação divergiram ligeiramente dos reais, sendo que a antena fabricada apresentou frequência de operação de $138,7 \mathrm{MHz}$ e perda de retorno de $-21,4 \mathrm{~dB}$, podendo ser utilizada para operar em múltiplas bandas como a de $3,1 \mathrm{GHz}, 5 \mathrm{GHz}$ e $5,9 \mathrm{GHz}$, ou seja, concluiu-se que a antena trabalha em frequência de micro-ondas.

\section{MATERIAIS E MÉTODOS}

A metodologia para avaliação do desempenho da antena planar dipolo curvado foi realizada primeiramente por meio de uma implementação numérica computacional no software Scilab versão 6.0. E em seguida pela simulação do projeto da antena, realizada no software ANSYS HFSS ${ }^{\circledR} 2019$, que utiliza o método dos elementos finitos para obter o comportamento dos parâmetros de funcionamento da antena. O HFSS ${ }^{\circledR}$ permite dentre diversas outras funcionalidades, realizar o design da antena, a análise em 2D e 3D dos diagramas de radiação e a plotagem de gráficos referentes a perda de retorno.

Os materiais adotados no projeto foram o cobre para a composição do patch e do plano terra e o FR4 (vibra de vidro) para o substrato. O modelo e as dimensões genéricas do projeto são visualizadas na Figura 1. O dimensionamento do dispositivo proposto foi baseado na faixa de frequência ISM 2,4GHz de operação, para aplicação na Indústria 4.0. Os parâmetros de entrada do projeto foram especificados como: (a) Frequência de ressonância $\left(f_{r}\right)$ de 2,45 GHz; (b) Permissividade relativa do substrato $\left(\varepsilon_{r}\right)$ de 4,4; (c) Espessura do substrato (h) de 1,56mm; (d) Impedância característica de entrada $\left(\mathrm{Z}_{\mathrm{o}}\right)$ de $50 \Omega$.

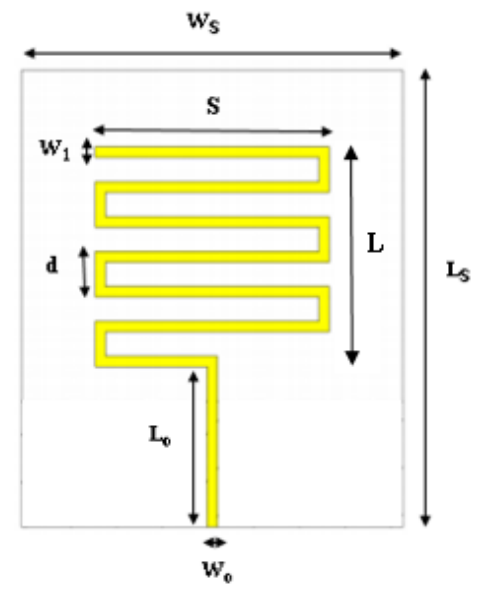

FIGURA 1. Modelo da antena meander line.

Um conjunto de equações foram necessárias para a realização da parametrização da antena com a obtenção das dimensões indicadas na Figura 1. A largura da linha de transmissão $\left(W_{o}\right)$ da antena, foi obtida pelas Equações (3) e (4) dispostas por [10]. Para o comprimento da linha de transmissão $\left(L_{o}\right)$, calculou-se baseado em [4] por meio das Equações (5) e (6), onde $\varepsilon_{e f}$ representa a permissividade efetiva.

$$
W_{o}=\frac{2 h}{\pi}\left[\left(B-1-\ln (2 B-1)+\frac{\varepsilon_{r}-1}{2 \varepsilon_{r}}\left\{\ln (B-1)+0.39-\frac{0.61}{\varepsilon_{r}}\right\}\right], \text { para } \frac{W_{o}}{h}>2\right.
$$




$$
\begin{aligned}
B & =\frac{337 \pi}{2 Z_{o} \sqrt{\varepsilon_{r}}} \\
L_{o} & =\frac{c}{4 f_{r} \sqrt{\varepsilon_{e f}}} \\
\varepsilon_{e f} & =\frac{\varepsilon_{r}+1}{2}+\frac{\varepsilon_{r}-1}{2}\left[1+12 \frac{h}{W_{o}}\right]^{\frac{-1}{2}}
\end{aligned}
$$

De acordo com [11], por se tratar de uma antena plana, leva-se em consideração o comprimento de onda guiado $\left(\lambda_{g}\right)$ dado em função da frequência de ressonância $\left(f_{r}\right)$ desejada e da permissividade relativa do substrato adotada:

$$
\lambda_{g}=\frac{c}{f_{r} \sqrt{\varepsilon_{e f}}}
$$

sendo que $c$ representa a velocidade da luz, aproximadamente $300000000 \mathrm{~m} / \mathrm{s}$.

A partir disso, as dimensões do patch da antena foram computadas e aproximadas em função do comprimento de onda calculado. O comprimento do patch da antena, referente a distância entre a linha de transmissão e a última linha de meandro, foi obtido por meio da Equação (8), enquanto que a largura do patch do meandro foi dada através da Equação (9). Além disso, a distância entre duas linhas de meandro (d) foi obtida pela Equação (10) e a largura da linha de meandro $\left(W_{1}\right)$ pela Equação (11).

$$
\begin{gathered}
L=0,70 \lambda_{g} \\
S=0,42 \lambda_{g} \\
d=0,16 \lambda_{g} \\
W_{1}=0,05 \lambda_{g}
\end{gathered}
$$

Com relação ao substrato, o comprimento $\left(L_{S}\right)$ foi calculado pela Equação $(12)$ e a largura $\left(W_{S}\right)$ por meio da Equação (13). Por fim, para o objetivo proposto não foi necessário realizar nenhuma redução de tamanho no plano de terra, sendo considerado as mesmas dimensões do substrato.

$$
\begin{gathered}
L_{S}=L+4 h+L_{o} \\
W_{S}=2 S+6 h
\end{gathered}
$$

\section{RESULTADOS E DISCUSSÕES}

O procedimento metodológico foi executado e as informações obtidas serão discutidas nesta seção. Na Figura 2 é mostrado o modelo em 3D da antena projetada. A simulação executada gerou o comportamento dos parâmetros da antena referente a perda de retorno, largura de banda e a distribuição de radiação de campo elétrico e campo magnético.

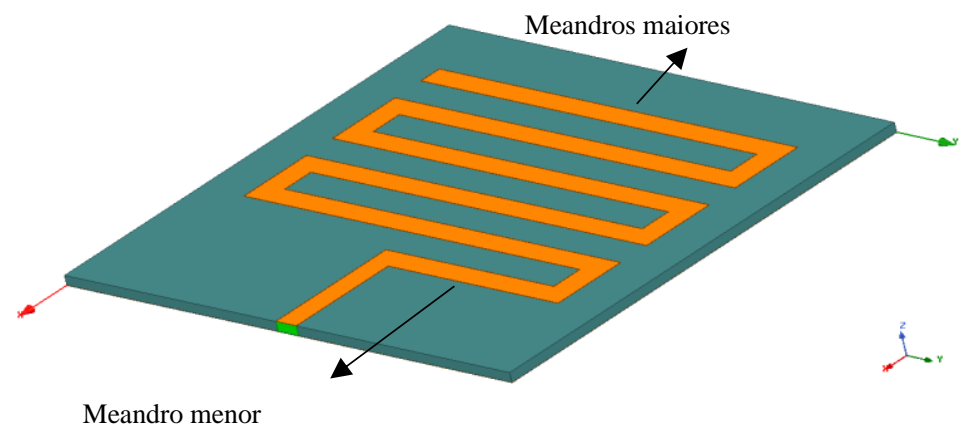

FIGURA 2. Design em 3D da estrutura da antena projetada no HFSS ${ }^{\circledR}$. 
Os valores obtidos para as dimensões da antena planar dipolo curvado estão dispostos na Tabela 1. É importante ressaltar que foi realizado alterações nos conceitos de parametrização da antena para que ocorresse a ressonância dentro da faixa de frequência para aplicação na banda ISM, conforme objetivo inicialmente proposto. As alterações foram feitas no comprimento das linhas de meandro horizontais do patch da antena, indicadas na Figura 2. O comprimento da primeira e menor linha de meandro horizontal recebeu o valor da variável $S$ calculada por meio da Equação (9) e para todas as cinco linhas maiores de meandro horizontais, adotou-se o dobro do valor de $S$. Essa mudança na largura do patch foi realizada no intuito de proporcionar a ressonância da antena na frequência dentro da faixa de operação desejada.

TABELA 1. Dimensões da antena meander line.

\begin{tabular}{cc}
\hline Parâmetros & Valor $(\mathrm{mm})$ \\
\hline$W_{o}$ & 3 \\
$L_{o}$ & 16,6 \\
$W_{1}$ & 3,3 \\
$L$ & 46,6 \\
$S$ & 27,9 \\
$d$ & 10,6 \\
$L_{S}$ & 69,4 \\
$W_{S}$ & 65,2 \\
\hline
\end{tabular}

\subsection{Perda de Retorno}

A Figura 3 ilustra a perda de retorno obtida na simulação, cujo intervalo de análise foi de $1,5 \mathrm{GHz}$ a 3,5GHz. Desse modo, observa-se que a antena meander line possui característica multibanda por ressoar em múltiplas frequências, identificadas pelos pontos $\mathrm{m} 1, \mathrm{~m} 2, \mathrm{~m} 3, \mathrm{~m} 4 \mathrm{e} \mathrm{m} 5$. Todas as frequências de retorno dentro da faixa de ressonância da antena são visualizadas na Tabela 2.

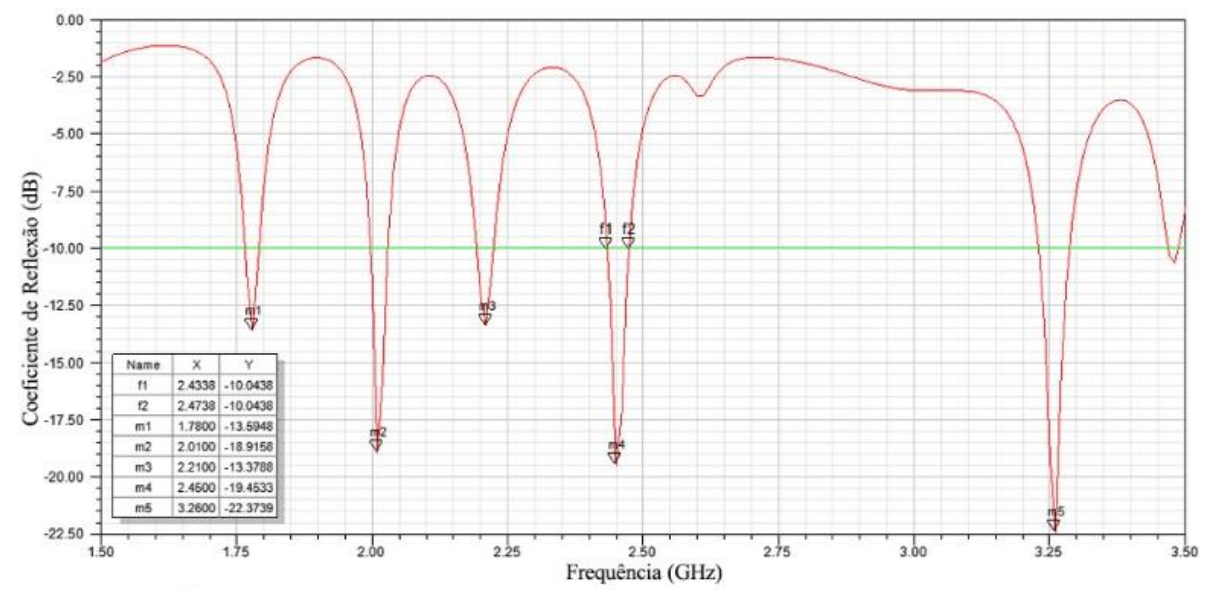

FIGURA 3. Variação da magnitude do coeficiente de reflexão em função da frequência.

TABELA 2. Frequências de operação da antena.

\begin{tabular}{cc}
\hline Frequência $(\mathrm{GHz})$ & $S(1,1)(\mathrm{dB})$ \\
\hline 1,78 & $-13,5948$ \\
2,01 & $-18,9158$ \\
2,21 & $-13,3788$ \\
2,45 & $-19,4533$ \\
3,26 & $-22,3739$ \\
\hline
\end{tabular}

Observa-se que a frequência de $2,45 \mathrm{GHz}$ é a única que se encontra dentro da banda ISM (2400 MHz até 2483,5 MHz), faixa de frequência de interesse deste artigo, portanto, a antena projetada atende aos prérequisitos de operação inicialmente propostos, qualificando-a de ser construída e aplicada nas redes Wireless na Indústria 4.0. Essa faixa de operação é melhor visualizada na Figura 4, em que o intervalo da simulação é reduzido. A partir das informações disponíveis, é extraída uma largura de banda igual a 40MHz, estabelecida 
pelo tamanho do intervalo entre a frequência inferior de $2,43 \mathrm{GHz}$ (ponto $f 1$ ) e a frequência superior de $2,47 \mathrm{GHz}$ (ponto $f 2$ ), em torno da frequência central de operação estudada, de 2,45GHz, caracterizando-a como uma antena de banda estreita. Também foi obtido uma largura de banda fracionária de 1,6\%. Vale destacar que o funcionamento da antena atende aos 11 canais para utilização do padrão IEEE 802.11b, voltado para as tecnologias de comunicação sem fio, se adequando totalmente a banda ISM e suas aplicações na Indústria 4.0.

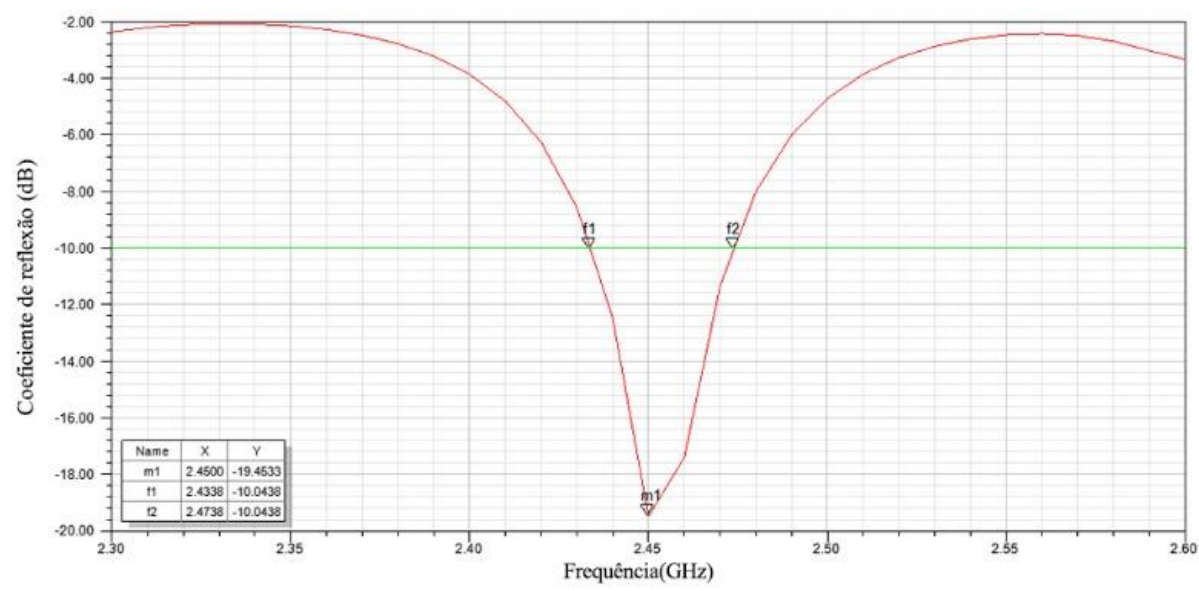

FIGURA 4. Variação da magnitude do coeficiente de reflexão em função da frequência.

\subsection{Diagrama de Radiação}

Os diagramas de radiação de campo magnético e de campo elétrico foram obtidos no HFSS®, como dispostos nas Figuras 5 e 6. Percebe-se que os diagramas são coerentes com a literatura e mostram que a distribuição de radiação do campo eletromagnético da antena meander line é proemenentemente omnidirecional, ou seja, transmite radiação em todas as direções ao seu redor referente a um plano. Na Figura 5 se observa que o sinal emitido é mais intenso na angulação de 0 a 180 graus referente ao plano E. O lóbulo secundário presente nesse intervalo é muito menor do que o primário e praticamente inexistente, logo, não interfere de modo significativo no desempenho da antena. A Figura 6 ilustra o padrão de radiação 3D, em que o ganho máximo alcançado é de aproximadamente -10dB.

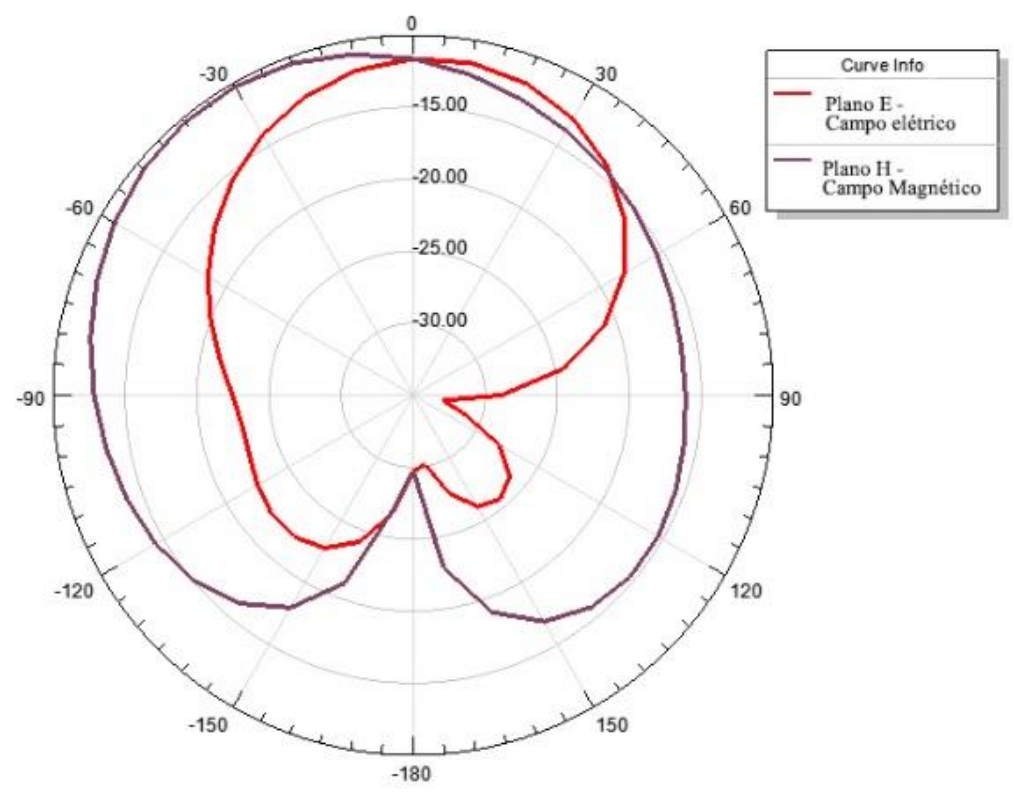

FIGURA 5. Diagrama de radiação 2D. 


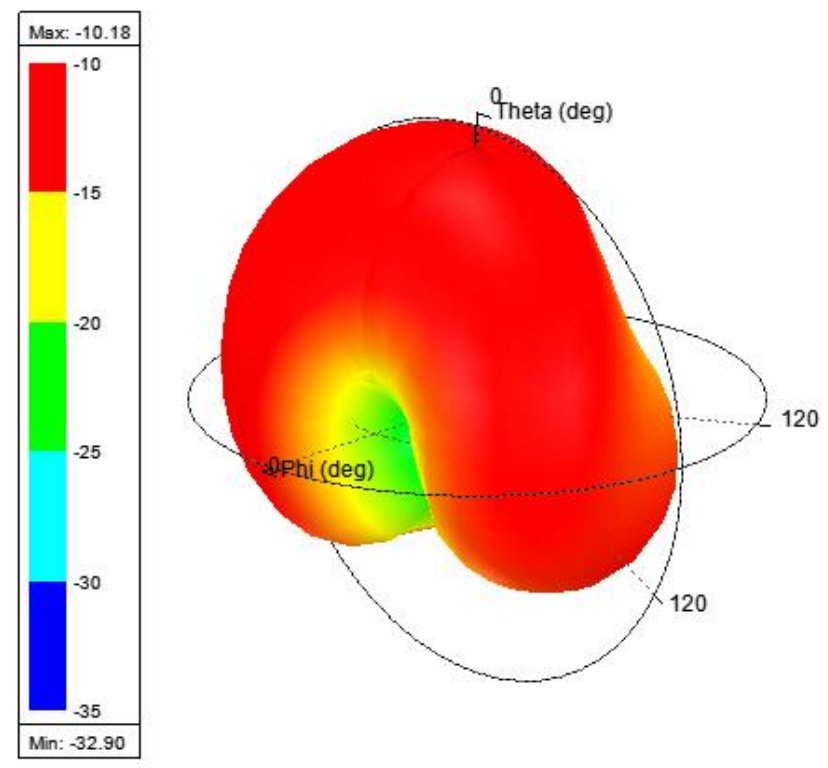

FIGURA 6. Diagrama de radiação 3D.

\section{CONCLUSÃO}

O desenvolvimento do presente artigo proporcionou uma análise de como as antenas planares dipolo curvado, meander line, são projetadas de acordo com a aplicação desejada, particularmente, dentro da faixa de frequência ISM 2,4GHz (2400 MHz até 2483,5 MHz), para utilização nas tecnologias presentes na Indústria 4.0, como a Internet of Things (IoT). Além disso, realizou-se um estudo de simulação no software ANSYS HFSS ${ }^{\circledR}$ 2019, avaliando o desempenho dos parâmetros da antena dimensionada e modelada. Com isso, observou-se o seu comportamento dentro da faixa ISM, validando que a antena projetada atende aos prérequisitos de alocação dos 11 canais do espectro ISM 2,4GHz com aplicações na comunicação da Indústria 4.0. Obteve-se uma boa percepção do comportamento dos parâmetros da antena, salientando que ela ressoou na frequência desejada de $2,45 \mathrm{GHz}$ para aplicações industriais, médicas e científicas, com valor adequado de perda de retorno. Além de apresentar uma largura de banda estreita, mostrando-se coerente para aplicação nas tecnologias voltadas a comunicação da Indústria 4.0.

Portanto, os resultados obtidos foram satisfatórios e estão em concordância com a literatura específica da área. A antena meander line é uma opção para aplicações na Indústria 4.0 e suas tecnologias, onde os aspectos de volume, baixo peso e adaptabilidade a superfícies são variáveis importantes. Além disso, também é desejável quando se busca ressonância em altas frequências, reduzir o espaço físico ocupado e baixo custo de fabricação. $\mathrm{O}$ estudo serve de embasamento para auxiliar em estudos posteriores e em novos projetos sobre as antenas planares dipolo curvado do tipo meander line ou similares.

\section{AGRADECIMENTOS}

Os autores agradecem à CAPES, CNPq, UFERSA, GEPEAT e à equipe do Laboratório de Micro-ondas da UFERSA. O presente trabalho foi realizado com apoio da Coordenação de Aperfeiçoamento de Pessoal de Nível Superior - Brasil (CAPES) - Código de Financiamento 001.

\section{REFERÊNCIAS}

[1] Magrani, E.; A Internet das Coisas, $1^{\circ}$ ed.; Rio de Janeiro: FGV Editora, Brasil, 2018; p. 1 - 192.

[2] Indústria 4.0 a moda a caminho do futuro. Disponível online: https://www.sebrae.com.br/Sebrae/Portal\%20Sebrae/UFs/RJ/Anexos/Industria\%204_0\%20\%20WEB.PDF (acesso em 15/06/2020).

[3] Alencar, M. S. de.; Queiroz, W. J. L. de. Ondas eletromagnéticas e teoria de antenas, 1rd ed.; São Paulo: Érica, Brasil, 2010; p. $1-225$.

[4] Balanis, C. A.; Antenna Theory: Analysis and Design, $3^{\circ}$ ed.; New Jersey: Wiley-interscience, EUA, 2005; p. $1-1047$. 
[5] Rashed, J.; Tai, C. T. A new class of resonant antennas. IEEE Trans. Antennas propagate 1991, vol. 39, 1428-1430

[6] Elamin, N. I. M.; Rahman, T. A.; Abdulrahman A. Y. New adjustable slot meander patch antenna for 4G handheld devices. IEEE Antennas and Wireless Propagation Letters 2013, vol. 12, 1077-1080.

[7] Freitas, E. F. de. Análise Paramétrica de Antenas de Linha Dobrada de Microfita (Microstrip Meander Line Antennas - Mmla). Dissertação, Universidade Federal Rural do Semi-árido, Mossoró, 2019.

[8] Manchanda, S.; Hatwar, M.; Abhishek, B. Design of Meander Line Antenna for Operating Frequency of 2. 5 GHz. International journal of computer applications (0975-8887) 2014, vol. 93, 33-36.

[9] Warsito, T.; Suprapto, Y. Desain Dan Fabrikasi Antena Mikrostrip Meander-Line Pada Frekuensi VHF (Very High Frequency) Untuk Komunikasi D2d. APPROACH: Jurnal Teknologi Penerbangan 2018, vol. 2, 29-34.

[10] Ma, M.; Deng, K. The Study and Implementation of Meander- Line Antenna for an Integrated Transceiver Design. Dissertation. University of Gävle ITB/ Eletronics, Suécia, 2010.

[11] Calla, O. P. N. et al. Empirical Relation for Designing the Meander Line Antenna. International Conference On Recent Advances In Microwave Theory And Applications. IEEE 2008, p.695-698. 